\title{
A Unified Model: Self-Assembly of Trimesic Acid on Gold
}

Yingchun Ye, Wei Sun, Yongfeng Wang, Xiang Shao, Xiaoguang Xu, Fang Cheng, Jianlong $\mathrm{Li}^{*}$, Kai Wu*

Beijing National Laboratory for Molecular Sciences, State Key Laboratory for Structural Chemistry of Unstable and Stable Species, College of Chemistry and Molecular Engineering, Peking University, Beijing 100871, China

E-mails: jlipku@pku.edu.cn; kaiwu@pku.edu.cn

\section{Supporting Information}

Figure S1 Periodic arrangements of the unit cells to form the hexagonal porous networks

Figure S2 Orientations of triangular TMA molecules in the unit cell for flower structure (HTMA-2).

Figure S3 Stability of the assembling structures across atomic steps on Au. 


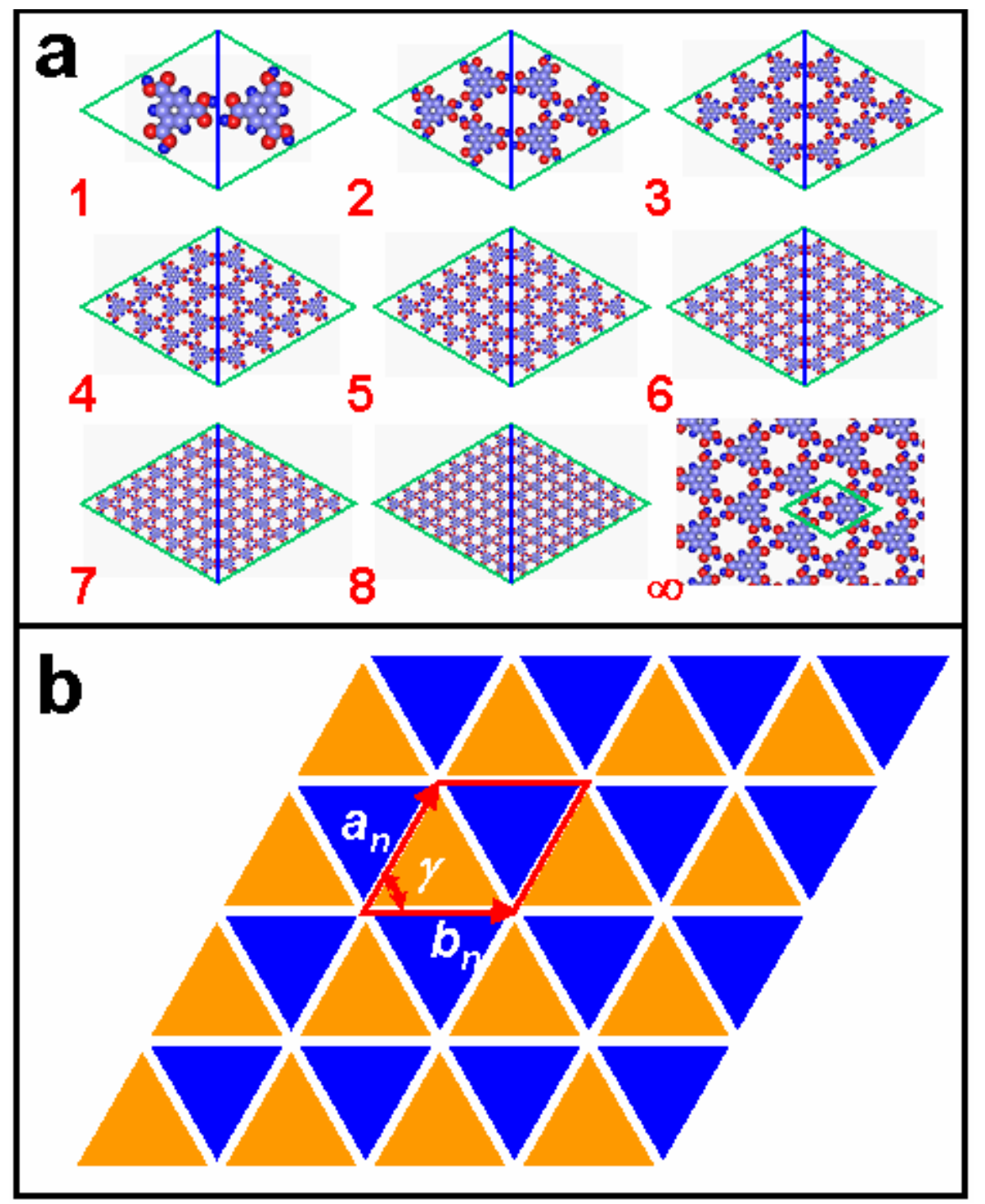

Figure S1 (a) Arrangements of the TMA molecules in the unit cells of the $\mathrm{H}_{\text {TMA-n }}(\mathrm{n}=1,2,3$, $4,5,6,7,8$ and $\infty$ ) structures. In the half unit cell (separated by the short diagonal of the unit cell), the number of the TMA molecules is $(1+2+3+\ldots+n)=n(n+1) / 2$ in $n$ rows for the

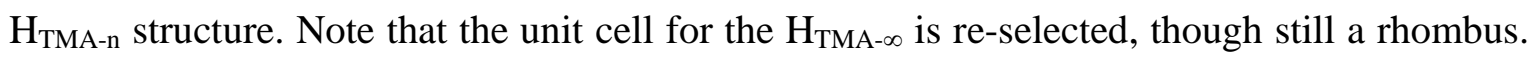
(b) Each rhombus unit cell in (a) can pack together to form a corresponding hexagonal porous network. Orange and blue equilateral triangles highlight the half unit cells. $a_{n}=b_{n}, \gamma=$ $60^{\circ}$. 

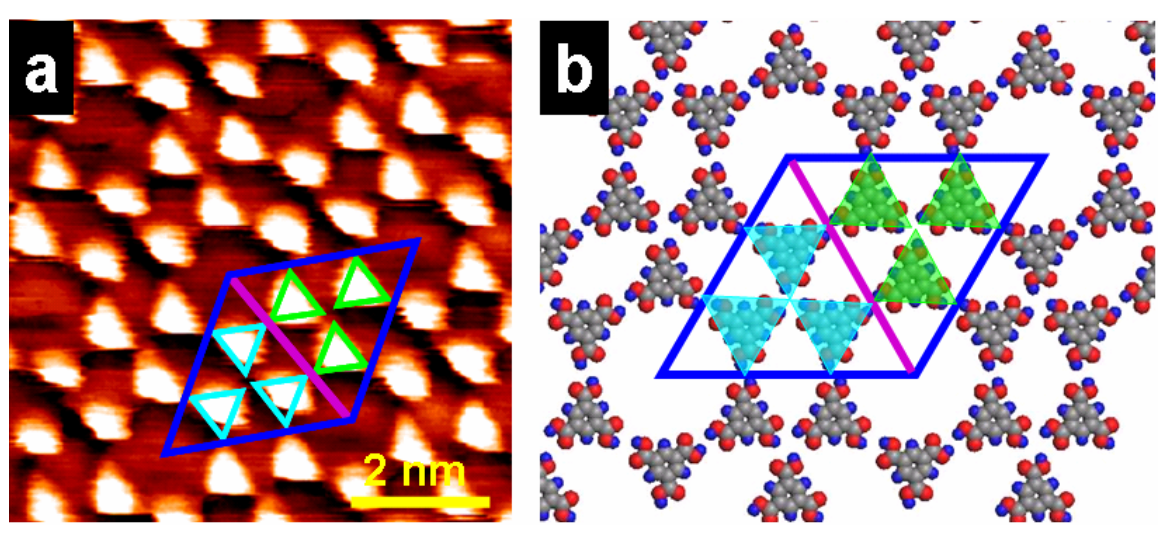

Figure S2 (a) STM image of the $\mathrm{H}_{\mathrm{TMA}-2}$ (flower) assembling structure. (b) Model of the $\mathrm{H}_{\text {TMA-2 }}$ structure. The parallelograms indicate the unit cell in which the triangular TMA molecules spatially orient [open and filled triangles in (a) and (b)]. 

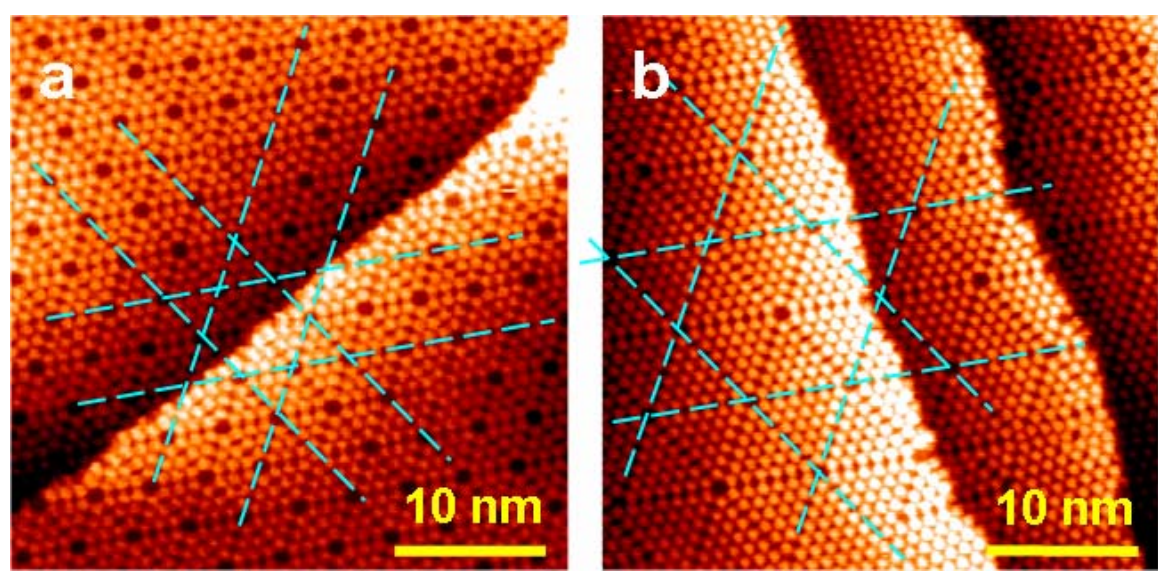

Figure S3 (a) STM image of the $\mathrm{H}_{\mathrm{TMA}-3}$ structure. (b) STM image of the $\mathrm{H}_{\text {TMA-6 }}$ structure. Note that both structures do not obviously deform across the steps on the gold substrate. 